\title{
Evaluation on Smart Growth of Sustainable Cities
}

Cheng Ming

\author{
Department of English, North China Electricity Power University, Baoding, 071000, China \\ email: 442867007@qq.com
}

Keywords: Smart Growth; City Project; Sustainable Development; Evaluation

\begin{abstract}
Based on the development idea of contemporary time, there exist large quantities of problems in the whole world during the process of urbanization. In order to improve living standards, sustainable development is believed as the prerequisite of the project and reconstruction of future cities. The objective was to develop a future smart growth program on the basis of the principles of smart growth in order to assess the growth of selected cities.
\end{abstract}

\section{Introduction}

Since the 21st century, men hold a brand new idea and standard about the project and construction of modern cities. Especially after the occurrence of global difficulties, such as environmental problems and population crisis, sustainable development has gradually been the priority of every country to build future cities, while the building standards of previous cities were opposed to the sustainable development principles in many areas. Thus, people constantly seek for novel city projects and developing ideas. The concept of smart growth originated from the 1990s [1]. After twenty years' exploration and practice, a few cities has already achieved desirable results, the success of which erects an ideal example in growth plan or developing model for those that are longing for changes. According to the existing successful cases, smart growth indeed solved numerous Gordian knots for modern cities, like traffic jam, ecological devastation, contradictions among people, etc. All sorts of the advantages prove it is a city project of modernization, sustainability and harmony.

This paper studied the three E's and ten principles for smart growth and considered that sustainable development should be the prerequisite for city project or reconstruction, together with maximum social fairness and economic prosperity. In addition, the effects of natural and non-natural factors on it were analyzed, like geographical position, opportunities of economic development, social structure and so on. At last, a careful testing was conducted.

\section{Smart Growth Evaluation Model}

Model Establishment. From Figure 1 which shows the Restrain Factors of Smart Growth, based on the three E's and ten principles for smart growth, we choose Environmentally Sustainable, Socially Equitable and Economically Prosperous as the restrictive conditions of total objective function $O$ (Smart Growth). Suitable strategies and principles in the ground level are adopted to give the following Table $1 O \mid C$ Judgment Matrix.

Hence, $C_{1}$ is assumed to be the most important in the restraint level, $C_{2}$ following and $C_{3}$ the last. Similarly, other factors can be accessible after calculation.

Table 2 respectively shows the ratio relationship among all the indicators of restraint level and strategy level, a preliminary conclusion can be draw. In the restraint level, the ratio of $C_{1}$ is the largest; in $C_{1}$ level, the ratio of $E S_{1}$ is the largest; in $C_{2}$ level, $S E_{1}$ is the largest; and in $C_{3}$ level, $E P_{1}$ is the largest.

From the eigenvalue and eigenvector of Table 2, Hierarchical total ranking can be shown in Table 3.

Figure 2 is the Hierarchical total ranking bar chart. From Figure 2, it can be known that all the Indicators of secondary level exert an influence on smart growth $O$. 


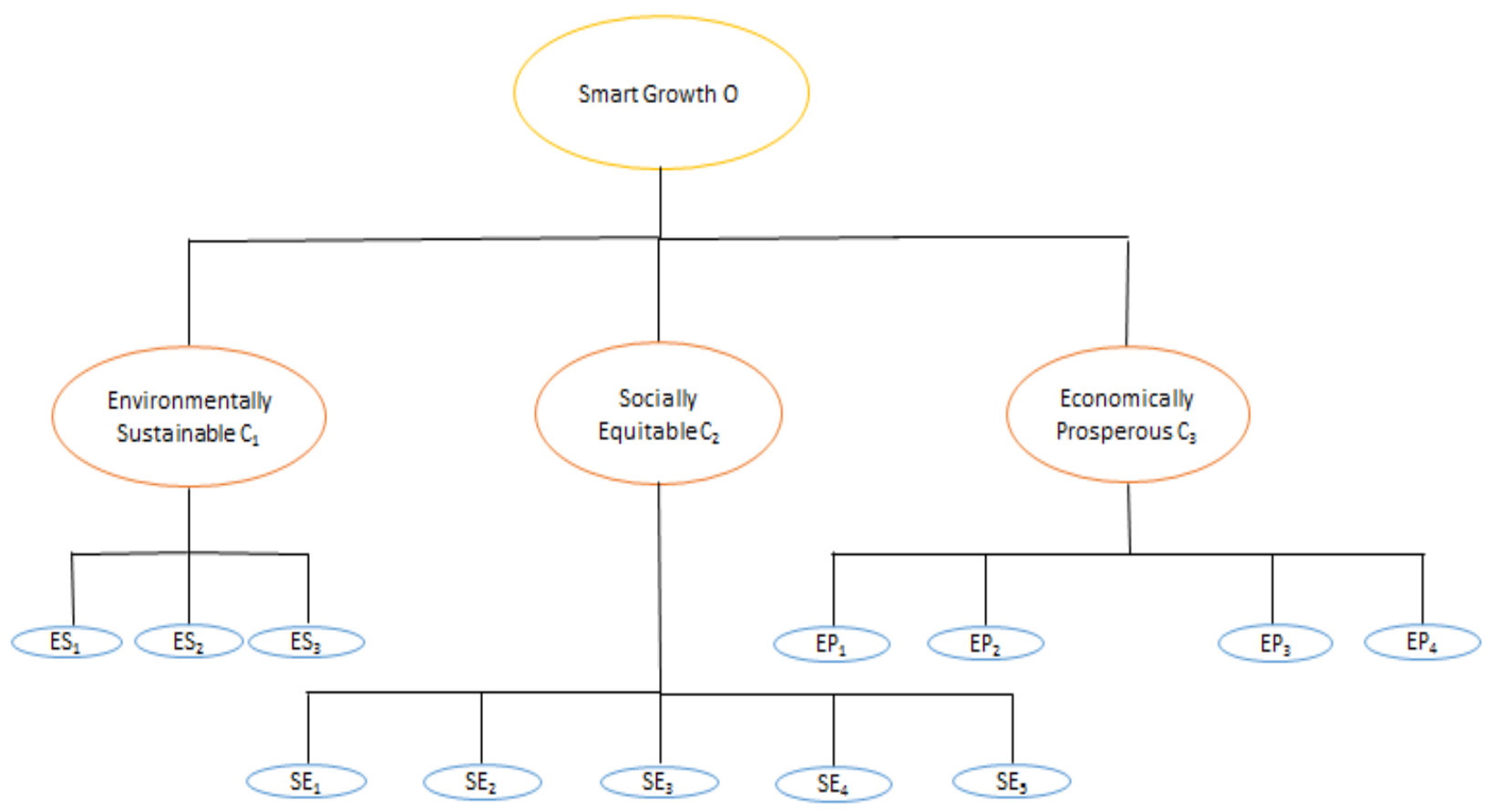

Fig. 1.Restrain Factors of Smart Growth

Table 1.O|C Judgment Matrix

\begin{tabular}{cccc}
\hline$O$ & $C_{1}$ & $C_{2}$ & $C_{3}$ \\
\hline$C_{1}$ & 1 & 3 & 4 \\
$C_{2}$ & $1 / 3$ & 1 & $4 / 3$ \\
$C_{3}$ & $1 / 4$ & $3 / 4$ & 1 \\
\hline
\end{tabular}

Table 2. Hierarchical single ranking conclusion

\begin{tabular}{ccccc}
\hline & $O \mid C$ & $C_{1} \mid E S$ & $C_{2} \mid S E$ & $C_{3} \mid E P$ \\
\hline$\lambda$ & 3 & 3 & 5 & 4 \\
\hline \multirow{4}{*}{$W$} & 0.6316 & 0.6 & 0.4225 & 0.3947 \\
& 0.2105 & 0.2 & 0.2113 & 0.3947 \\
& 0.1579 & 0.2 & 0.1408 & 0.1316 \\
& no & no & 0.1408 & 0.0789 \\
& no & no & 0.0845 & no \\
\hline
\end{tabular}

Table 3. Hierarchical total ranking

\begin{tabular}{ccccc}
\hline \multirow{2}{*}{$A$} & $C_{1}$ & $C_{2}$ & $C_{3}$ & \multirow{2}{*}{ Total Order Value } \\
\cline { 2 - 4 } & 0.6316 & 0.2105 & 0.1579 & \\
\hline$E S_{1}$ & 0.6 & no & no & 0.37896 \\
$E S_{2}$ & 0.2 & no & no & 0.12632 \\
$E S_{3}$ & 0.2 & no & no & 0.12632 \\
$S E_{1}$ & no & 0.4225 & no & 0.08894 \\
$S E_{2}$ & no & 0.2113 & no & 0.04448 \\
$S E_{3}$ & no & 0.1408 & no & 0.02964 \\
$S E_{4}$ & no & 0.1408 & no & 0.02964 \\
$S E_{5}$ & no & 0.0845 & no & 0.01779 \\
$E P_{1}$ & no & no & 0.3947 & 0.06232 \\
$E P_{2}$ & no & no & 0.3947 & 0.06232 \\
$E P_{3}$ & no & no & 0.1316 & 0.02078 \\
$E P_{4}$ & no & no & 0.0789 & 0.01246 \\
\hline
\end{tabular}




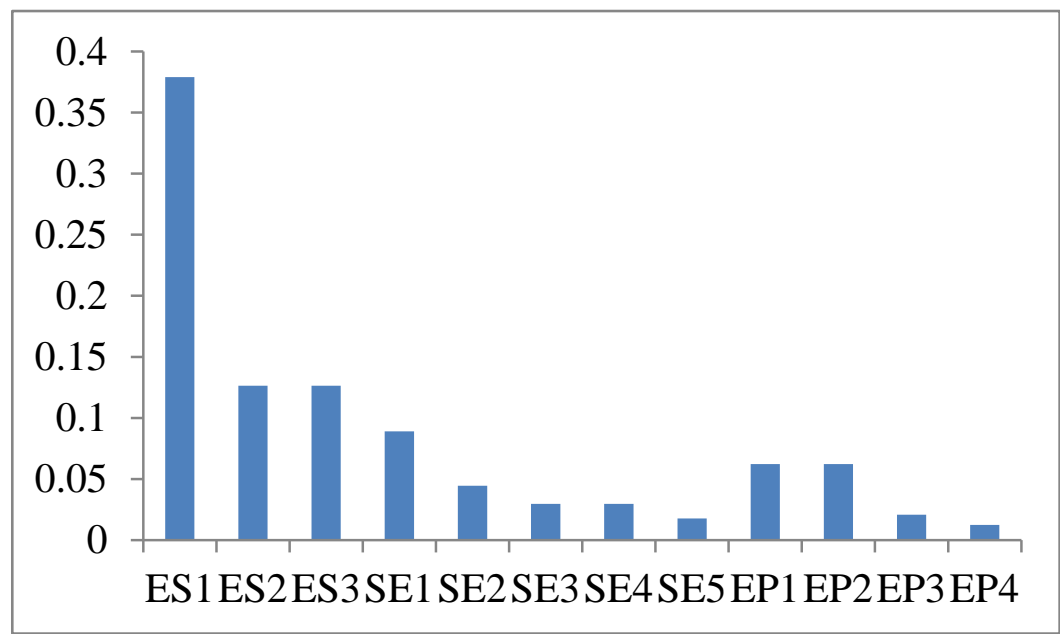

Fig. 2 Hierarchical total ranking bar chart

Results. According to the results above, it can be obtained the relationship between smart growth $O$ and all the evaluation indicators are quantified and the following equation is derived to demonstrate the quantitative relationship between smart growth $O$ and all sub-indexes.

$$
\begin{aligned}
O=0.37896 E & S_{1}+0.12632 E S_{2}+0.12632 E S_{3}+0.08894 S E_{1}+0.04448 S E_{2}+0.02964 S E_{3} \\
& +0.02964 S E_{4}+0.01779 S E_{5}+0.06232 E P_{1}+0.06232 E P_{2}+0.02078 E P_{3} \\
& +0.01246 E P_{4}
\end{aligned}
$$

The relevant data of selected cities are put into the above equation, so a quantized value can be derived that can quantify the extent to smart growth. Then it is contrasted with the planned standard value and the relative value of its grade of success can be shown as Table 4 .

Table 4. Standard of Smart Growth

\begin{tabular}{ccccc}
\hline$O$ & $<5 \%$ & $5 \% \sim 10 \%$ & $10 \% \sim 15 \%$ & $>15 \%$ \\
Level & $D$ & $C$ & $B$ & $A$ \\
\hline
\end{tabular}

\section{Establishment of Future Smart Growth Program}

The refinement index of standard cities is combined with their actual situation and then respectively make the future growth plans for Karamay City and City of Manchester.

Karamay City. The city of Karamay is located in the northwestern part of Xinjiang's Junggar Basin [2]. It is a new, modern industrialized city with powerful economic strength. It is abundant in gas and oil resources and has already built a complete industrial chain in the oil and petroleum industries [3]. In line with the three E's and ten principles for smart growth, a future growth program for Karamay City is shown as Table 5.

City of Manchester. Manchester lies in a bowl-shaped land area bordered to the north and east by the Pennines, a mountain chain that runs the length of northern England, and to the south by the Cheshire Plain. The River Mersey flows through the south of Manchester [4]. Manchester's geographic features were highly influential in its early development as the world's first industrial city. These features are its climate, its proximity to a seaport at Liverpool, the availability of water power from its rivers, and its nearby coal reserves [5]. In terms of its location and economic development, a future growth program for Manchester is illustrated as Table 6.

\section{Evaluation of Future Growth Program}

According to the above equation of Smart Growth Evaluation Model, we can calculate and derive as follows.

Smart growth index of Karamay City $\boldsymbol{O}=\mathbf{9 . 3 \%}$. Compared with the original plan made by Government, our future growth program is largely improved. However, it is still of medium level, for it does not achieve smart growth, which is directly related to the geographical location of 
Karamay-in the desert. In the view of smart growth index $O$, the plan we devised is temporarily incapable of making the target city realize perfect smart growth, yet the development of a city cannot accomplish at one stroke, but project step by step in accordance with the actual situation. From this perspective, our program is feasible.

Smart growth index of Manchester $\boldsymbol{O}=\mathbf{1 0 . 6 \%}$. The future growth program of Manchester, which reflects the real situation there, is relatively successful, since it is designed in line with the ten principles of smart growth, geographical position, opportunities of economic development and the original growth plan made by Government. If it can be carried on in this way, it will achieve $A$ level of smart growth.

Table 5. Karamay City Future Growth Program

\begin{tabular}{cc}
\hline Percentage of urban environmental construction costs accounting for $G R P E S_{1}$ & $16 \%$ \\
Percentage of natural ecological area $E S_{2}$ & $0.00 \%$ \\
Land utilization ratio $E S_{3}$ & $0.00 \%$ \\
Per capita income of urban residents $S E_{1}$ & $9.30 \%$ \\
Per capita income of rural residents $S E_{2}$ & $12.50 \%$ \\
Urbanization rate $S E_{3}$ & $6.20 \%$ \\
Unemployment rate $S E_{4}$ & $2.80 \%$ \\
Natural population growth rate $S E_{5}$ & $3.80 \%$ \\
Gross Regional Product $E P_{1}$ & $9 \%$ \\
Total investment in fixed assets $E P_{2}$ & $13 \%$ \\
Local fiscal revenue $E P_{3}$ & $15.40 \%$ \\
Retail sales of social consuming goods $E P_{4}$ & $11.10 \%$ \\
\hline Table 6. City of Manchester Future $G r o w t h$ Program & \\
\hline Percentage of urban environmental construction costs accounting for $G R P \quad E S_{1}$ & $16.30 \%$ \\
Percentage of natural ecological area $E S_{2}$ & $0.50 \%$ \\
Land utilization ratio $E S_{3}$ & $0.01 \%$ \\
Per capita income of urban residents $S E_{1}$ & $16.10 \%$ \\
Per capita income of rural residents $S E_{2}$ & $13.60 \%$ \\
Urbanization rate $S E_{3}$ & $2.30 \%$ \\
Unemployment rate $S E_{4}$ & $4.80 \%$ \\
Natural population growth rate $S E_{5}$ & $5.30 \%$ \\
Gross Regional Product $E P_{1}$ & $14 \%$ \\
Total investment in fixed assets $E P_{2}$ & $16 \%$ \\
Local fiscal revenue $E P_{3}$ & $13.20 \%$ \\
Retail sales of social consuming goods $E P_{4}$ & $13 \%$ \\
\hline
\end{tabular}

\section{Testing Results}

Here the paper chose Beijing and Chongqing city of China to conduct sensitivity analysis to determine the extent to which the model depends upon those influencing factors.

It can be calculated that Smart growth index of Beijing $O=9.26 \%$ and Smart growth index of Chongqing $O=8.82 \%$ are both of smart growth moderate level shown as Table 7 .

Contrast. Beijing is the capital of the People's Republic of China. A city combining both modern and traditional architecture, Beijing is an ever-changing megacity rich in history but also truly modern, exemplified in its extraordinary global influence on politics, education, history, culture, language, architecture, art, innovation and technology, yet the industrial development is uncoordinated [6]. Thus, the smart growth index of Beijing is described as moderate. Chongqing is a major city in the southwest of China. It is situated at the transitional area between the Qinghai-Tibet Plateau and the plain on the middle and lower reaches of the Yangtze River in the sub-tropical climate zone often swept by moist monsoons [7]. The utilization ratio of the underground is very high, but since Chongqing city sprawls excessively, we define smart growth index of Chongqing as moderate. 
Table 7 Sensitivity Analysis

\begin{tabular}{cccc}
\hline Evaluation Value & Ratio & Beijing & Chongqing \\
\hline$E S_{1}$ & 0.37896 & $18.00 \%$ & $14.90 \%$ \\
$E S_{2}$ & 0.12632 & $0.02 \%$ & $0.87 \%$ \\
$E S_{3}$ & 0.12632 & $0.04 \%$ & $0.02 \%$ \\
$S E_{1}$ & 0.08894 & $8.00 \%$ & $8.30 \%$ \\
$S E_{2}$ & 0.04448 & $8.00 \%$ & $10.70 \%$ \\
$S E_{3}$ & 0.02964 & $2.20 \%$ & $2.20 \%$ \\
$S E_{4}$ & 0.02964 & $3.50 \%$ & $1.25 \%$ \\
$S E_{5}$ & 0.01779 & $3.80 \%$ & $2.00 \%$ \\
$E P_{1}$ & 0.06232 & $8.00 \%$ & $10.70 \%$ \\
$E P_{2}$ & 0.06232 & $9.00 \%$ & $12.10 \%$ \\
$E P_{3}$ & 0.02078 & $9.00 \%$ & $10.60 \%$ \\
$E P_{4}$ & 0.01246 & $7.30 \%$ & $13.20 \%$ \\
$\boldsymbol{O}$ & & $\mathbf{9 . 2 6 \%}$ & $\mathbf{8 . 8 2 \%}$ \\
\hline
\end{tabular}

\section{Conclusion}

The task was to develop a future smart growth program on the basis of the principles of smart growth in order to assess the growth of selected cities. Finally, it can be safely drawn a conclusion from the evaluation system, which is shown as follows.

Though smart growth enables to solve many problems during the process of urbanization, urban population is much likely to increase swiftly due to the construction of livable cities. Therefore, considerable population will bring about huge stress for society. In order to support this level of growth, a series of measures should be taken, like investment in education, improving the population quality, taking advantage of compact building design and raising the land utilization ratio, etc.

\section{Acknowledgement}

In this paper, the research was based on the previous investigation of others and the official online information.

\section{References}

[1] https://en.wikipedia.org/wiki/Smart_growth

[2] http://www.chinadaily.com.cn/m/xinjiang/karamay/2015-04/09/content_20403235.htm

[3] https://en.wikipedia.org/wiki/Karamay

[4] https://en.wikipedia.org/wiki/Manchester

[5] https://en.wikipedia.org/wiki/Economy_of_Manchester

[6] https://en.wikipedia.org/wiki/Beijing

[7] https://en.wikipedia.org/wiki/Chongqing 